\title{
1 Alterations in SARS-CoV-2 Omicron and Delta 2 peptides presentation by HLA molecules
}

Stepan Nersisyan ${ }^{1,2,3}$, Anton Zhiyanov², Maria Zakharova², Irina Ishina ${ }^{2}$, Inna Kurbatskaia ${ }^{2}$, Azad Mamedov' ${ }^{2}$, Alexey Galatenko ${ }^{1,4}$, Maxim Shkurnikov ${ }^{1}$, Alexander Gabibov ${ }^{2}$, Alexander 6 Tonevitsky ${ }^{1,2,3}$

${ }^{1}$ Faculty of Biology and Biotechnology, HSE University, Moscow, Russia

${ }^{2}$ Shemyakin-Ovchinnikov Institute of Bioorganic Chemistry, Russian Academy of Sciences, Moscow, Russia

${ }^{3}$ SRC Bioclinicum, Moscow, Russia

${ }^{4}$ Faculty of Mechanics and Mathematics, Lomonosov Moscow State University, Moscow, Russia

Corresponding Author: Maxim Shkurnikov ${ }^{1}$

Vavilova str. 7, Moscow, Russia, 117312, Russia

Email address: $\underline{\text { mshkurnikov@ @hse.ru }}$

\section{Abstract}

The T-cell immune response is a major determinant of effective SARS-CoV-2 clearance. Here, using the recently developed T-CoV bioinformatics pipeline (https://t-cov.hse.ru) we analyzed the peculiarities of the viral peptide presentation for the Omicron, Delta and Wuhan variants of SARS-CoV-2. First, we showed the absence of significant differences in the presentation of SARS-CoV-2-derived peptides by the most frequent HLA class I/II alleles and the corresponding HLA haplotypes. Then, the analysis was limited to the set of peptides originating from the Spike proteins of the considered SARS-CoV-2 variants. The major finding was the destructive effect of the Omicron mutations on PINLVRDLPQGFSAL peptide, which was the only tight binder from the Spike protein for HLA-DRB1*03:01 allele and some associated haplotypes. Specifically, we predicted a dramatical decline in binding affinity of HLA-DRB $1 * 03: 01$ and this peptide after N211 deletion, L212I substitution and EPE 212-214 insertion. The computational prediction was experimentally validated by ELISA with the use of corresponding thioredoxin-fused peptides and recombinant HLA-DR molecules. Another finding was the significant reduction in the number of tightly binding Spike peptides for HLA-B*07:02 HLA class I allele (both for Omicron and Delta variants). Overall, the majority of HLA alleles and haplotypes was not significantly affected by the mutations, suggesting the maintenance of effective T-cell immunity against the Omicron and Delta variants. Finally, we introduced the Omicron variant to T-CoV portal and added the functionality of haplotype-level analysis to it. 


\section{Introduction}

T-cell immune response plays a pivotal role in the pathogenesis of COVID-19 (Sekine et al., 2020; Nelde et al., 2021; Shkurnikov et al., 2021). Cytotoxic (CD8) T-cells become activated through recognition of viral peptides presented by HLA class I molecules on the surface of antigen-presenting cells (APCs). The same mechanism based on recognition of HLA-I/peptide complex is further used to identify and destroy infected cells. Unlike cytotoxic T-cells, helper (CD4) T-cells become activated through interaction between their T-cell receptors (TCR) and viral peptides presented by HLA class II proteins. One of the main effector functions of helper Tcells consists in delivering the second activation signal to B-cells, which is necessary for the initiation of antibody production (Kumar, Connors \& Farber, 2018).

Recently emerged SARS-CoV-2 variants effectively escape neutralization by antibodies directed to the Spike protein of the base Wuhan variant. According to Nextstrain project, at the beginning of February 2022 most of new COVID-19 cases were driven by Omicron and Delta variants. While replication and antibody-mediated neutralization of these variants were studied extensively (Shiehzadegan et al., 2021; Cameroni et al., 2021; Zhao et al., 2022), the role of Tcell immune response and possibility of T-cell immunity evasion are to be uncovered. In several recent studies preservation of robust T-cell immunity against the Omicron variant was suggested (GeurtsvanKessel et al., 2021; Keeton et al., 2021; May et al., 2021; Liu et al., 2022; Mazzoni et al., 2022). However, a more elaborative analysis should be conducted to address the populationlevel diversity of HLA molecules.

We recently developed T-cell COVID-19 Atlas (T-CoV) - the computational pipeline and web portal for evaluation of impact of SARS-CoV-2 mutations on HLA-peptide interactions (Nersisyan et al., 2022). Here we used T-CoV to compare viral peptide presentation for the three variants: Wuhan, Delta and Omicron. Since the major part of the existing vaccines are based on the Spike protein of the reference variant (Kyriakidis et al., 2021), the comparisons were separately conducted for the whole virus and Spike protein peptidomes. At the beginning, the analysis was performed on a single allele-level; 64 HLA class I (HLA-A, HLA-B, HLA-C) and 105 HLA class II (HLA-DR, HLA-DQ, HLA-DP) abundant alleles were screened. Then, the most relevant findings (differential HLA-peptide interactions) were validated experimentally with ELISA. Next, the considered alleles were combined into the theoretical library of all possible haplotypes, and the peptide presentation analysis was conducted at the level of haplotypes. Finally, Allele Frequency Net Database was utilized to highlight the most frequent haplotypes with altered Omicron/Delta peptide presentation (Gonzalez-Galarza et al., 2020). The workflow of the study is presented in Figure 1.

Aside from the conducted analysis, we made two updates to T-CoV portal. First, we added the Omicron variant to the list of available variants. Second, the haplotype-level analysis was introduced to the portal in a form of user-friendly web tool (https://t-cov.hse.ru/haplotypes). 


\section{Materials \& Methods}

\section{Bioinformatics analysis of HLA/peptide interactions}

Protein sequences of SARS-CoV-2 variants were obtained from GISAID (Elbe \& Buckland-

Merrett, 2017):

- EPI_ISL_402125 (Wuhan);

- EPI_ISL_1663516 (Delta);

- EPI_ISL_6699752 (Omicron BA.1).

T-CoV pipeline was executed for the analysis of HLA/peptide interactions (Nersisyan et al., 2022). Briefly, binding affinities of all viral peptides and 169 frequent HLA class I/II molecules were predicted with netMHCpan 4.1 and netMHCIIpan 4.0 (Reynisson et al., 2020). Then, predicted affinities were compared between the reference Wuhan variant and Omicron/Delta. HLA/peptide pairs whose affinities were altered by at least two folds were used in the downstream analysis.

\section{HLA-DRB1*03:01/peptide binding experiments}

HLA-DR/peptide binding experiments were conducted as we previously described (Mamedov et al., 2020). Briefly, the genetic constructions for recombinant HLA-DR $\alpha$ and $\beta$ (HLA-

DRB1*03:01) chains with CLIP expression in in HEK293F suspension cells were created based on pFUSE vector encoding constant fragment of human immunoglobulin heavy chain ( $\mathrm{Fc}$ ). CLIP (PVSKMRMATPLLMQA) was covalently attached with the linker with a thrombin site at the $\mathrm{N}$-terminus of $\beta$ chain.

Five thioredoxin-fused 15-mer peptides were constructed to experimentally validate netMHCIIpan-predicted differences in HLA-DRB1*03:01 binding because of the Omicron mutations:

- PINLVRDLPQGFSAL (the reference Wuhan/Delta peptide);

- TPIIVREPEDLPQGF, IIVREPEDLPQGFSA, VREPEDLPQGFSALE, EPEDLPQGFSALEPL (four Omicron peptides with different shifts compared to the reference sequence).

The substrate construct, carrying only thioredoxin with the linker, was used as a negative control. Thioredoxin-fused peptides were chemically biotinylated with EZ-Link Sulfo-NHS-LC-biotin (Thermo Fisher Scientific).

Biotinylated and thioredoxin-fused peptides $(750 \mathrm{nM})$ were incubated overnight at $37^{\circ} \mathrm{C}$ in PBS in $50 \mu \mathrm{l}$ with HLA-DR (HLA-DRB1*03:01, 150nM). DR/peptide complexes were then captured on the ELISA plate with immobilized L243 $\mathrm{mAb}(5 \mu \mathrm{g} / \mathrm{ml}$ in PBS) and blocked with $1 \%$ milk in 
phosphoric acid (50 $\mu \mathrm{l})$. ELISA was performed using standard protocol (https://www.abcam.com/protocols/sandwich-elisa-protocol-1).

\section{HLA allele and haplotype frequency analysis}

HLA allele and haplotype frequencies were downloaded from Allele Frequency Net Database (Gonzalez-Galarza et al., 2020) for the following regions: Europe, North America, North-East Asia, South Asia, South and Central America, South-East Asia and Western Asia. For HLA class I haplotypes three genes were selected: HLA-A, HLA-B, HLA-C. For HLA class II three haplotype pools were analyzed separately:

- HLA-DQA1, HLA-DQB1;

In addition to the worldwide frequency data, we used our previously described dataset of 428 volunteers to assess the HLA alleles distribution in Moscow, Russia (Shkurnikov et al., 2021). Briefly, HLA-A, HLA-B, HLA-C, HLA-DRB1, HLA-DQB1 genes were sequenced with the MiSeq platform (Illumina, San Diego, CA, USA) using reagent kit HLA-Expert (DNATechnology LLC, Moscow, Russia). Frequencies of HLA-A/B/C and HLA-DRB1/DQB1 haplotypes were inferred with Hapl-o-Mat v1.1; the expectation-maximization algorithm with default settings was used (Schäfer, Schmidt \& Sauter, 2017).

\section{Results}

\section{Design of the allele-level analysis}

With the use of T-CoV database we obtained the lists of viral peptides which were predicted to be tight binders (affinity $\leq 50 \mathrm{nM}$ ) for the worldwide prevalent HLA class I and class II alleles. The analysis was conducted for three SARS-CoV-2 variants: Wuhan, Delta and Omicron. Since the immune system of many individuals was exposed only to Spike protein of the reference Wuhan variant (Spike protein-based vaccination), we used two peptide pools: the whole peptidome of the virus and the peptidome of the Spike protein.

First, we performed individual allele-level analysis. Given the numbers of tightly binding peptides for the fixed HLA allele and three SARS-CoV-2 variants, we calculated the differences in the numbers of tight binders of the Delta and Omicron variants relative to the Wuhan virus (Table S1). For example, -100\% difference indicated complete vanishing of all tightly binding Wuhan virus peptides, while $100 \%$ increase indicated doubling the number of peptides. Note that denominators included the number of tight binders in all viral proteins or solely Spike protein 
Ten HLA class I alleles had altered presentation of peptides from the Spike proteins of the Omicron and Delta variants virus: a single peptide from both Delta and Omicron variants (FPLTSFGPL) originating from the NSP12 protein became tight binder for HLA-B*35:03 because of P323L substitution (50\% relative increase in the number of tight binders).

Much more alleles (ten) demonstrated significantly altered peptide presentation $(\leq-25 \%$ or $\geq$ $25 \%$ ) during the analysis of the Spike protein peptides (Figure 2). Eight alleles were found in the context of the Omicron variant, and only three alleles showed differential presentation of the Delta peptides (HLA-B*07:02 allele was marked for both variants). The results were also skewed in the direction of enhanced peptide presentation (7/10 alleles).

Three alleles, HLA-B*07:02, HLA-B*27:05 and HLA-A*32:01, had more than 50\% difference in the number of presented Omicron/Delta peptides. The impact of the Delta and Omicron mutations on the peptide presentation was the same for HLA-B*07:02 allele: SPRRARSVA and three surrounding peptides lost their binding affinity because of P681H mutation. Next, L452R substitution in the Delta variant led to the emergence of three novel tight binders (YRYRLFRK, YRYRLFRKSNL, YRYRLFRKSNLK) for HLA-B*27:05. Finally, two tight binders for HLAA*32:01 appeared because of the mutations in the Omicron variant: VLYNLAPFF (S371L, S373P, S735F) and RSYSFRPTY (Q493R, G496S, Q498R, N501Y).

\section{HLA-DRB1*03:01 lost all tight binders from the Spike protein due to the mutations} in the Omicron variant

Not a single HLA class II allele passed $25 \%$ difference threshold when the analysis was were highlighted: HLA-DRB 1*03:01 for the Omicron variant and HLADPA1*01:03/DPB1*03:01 for the Delta variant.

The striking observation consisted in fact that all predicted tight binders from the Spike protein of the Wuhan and Delta variants for HLA-DRB $1 * 03: 01$ allele vanished because of the Omicron mutations. There were several tightly binding peptides for HLA-DRB1*03:01 centered around PINLVRDLPQGFSAL peptide with the nine amino acid core LVRDLPQGF (binding affinity 27 $\mathrm{nM}$ ). The Omicron variant had two mutations within the peptide core (L212I substitution, EPE 212-214 insertion) and N211 deletion in the peptide flank (Figure 3A). According to the predictions obtained by netMHCIIpan, all corresponding Ocmicron peptides became non-binders for HLA-DRB $1 * 03: 01$ allele (predicted affinity $>5000 \mathrm{nM}$ ). 
In order to experimentally validate the computational predictions, biotinylated and thioredoxinfused reference PINLVRDLPQGFSAL peptide from the Wuhan/Delta Spike proteins, as well as four matching Omicron peptides were constructed. Then, the constructed peptides and recombinant HLA-DR molecules (HLA-DRB1*03:01) were incubated together overnight, and DR/peptide complexes were captured on the ELISA plate. In full accordance with the bioinformatics predictions, the reference peptide bound HLA-DRB1*03:01, while the corresponding Omicron peptides were not forming complexes with the same HLA-DR receptors (Figure 3B).

The importance of the finding is emphasized by the high frequency of HLA-DRB1*03:01 allele in some regions, including 8.9\% in Europe and 10.0\% in Moscow, Russia. As we have already mentioned, presentation of the Omicron peptides by HLA-DRB1*03:01 was significantly different from the Wuhan variant only for the Spike protein, since there were several conserved tight binders in other proteins (N, NSP2, NSP3, NSP4, NSP5, NSP8, NSP12, NSP13, NSP14). Thus, the reduced presentation efficiency of HLA-DRB1*03:01 could possibly affect only individuals vaccinated with Spike protein-based vaccines.

There was another HLA class II allele with differential peptide presentation. Specifically, P681R mutation in the Delta variant slightly strengthened binding affinity of two peptides: YQTQTNSPRRARSVASQSII (84 $\mathrm{nM}$ to $40 \mathrm{nM}$ ) and QTQTNSPRRARSVASQSIIA (66 nM to $33 \mathrm{nM})$.

The Omicron and Delta mutations altered peptide presentation efficiency for several HLA class I and II haplotypes presentation. At the same time, each individual carries multiple alleles at once, including two parental alleles of HLA class I genes (HLA-A, HLA-B, HLA-C) and HLA class II genes (HLADRB1, HLA-DQA1, HLA-DQB1, HLA-DPA1, HLA-DPB1). All these genes are closely linked, and a whole HLA haplotype is inherited from each patient (Choo, 2007). Given that, a single "weakened" allele in a set of "strong" alleles will not affect much total peptide presentation by individual's HLA molecules set. To assess whether HLA haplotypes with significantly different presentation of Omicron/Delta peptidomes exist, two sets of haplotypes were analyzed:

1. Theoretical library of all possible haplotypes composed of HLA alleles under consideration.

2. Library of the most frequent HLA haplotypes in several populations.

We also updated our T-CoV portal to allow users to perform the analysis with their set of HLA alleles (Figure 4). 
datasets. Because each HLA-DPB1 and HLA-DQB1 allele is closely linked with only few alpha chain variants, we then manually adjusted the results for HLA-DPA1/DPB1 and HLADQA1/DQB1 links. Omicron/Delta peptide presentation at the level of the whole SARS-CoV-2 proteome (Table S2). As expected, all these genotypes contained HLA-B*35:03 allele, which was the only selected entry in the individual allele analysis. The single haplotype (A*25:01-B*35:03-C*04:01) was marked as frequent in Europe (frequency $=0.02 \%$ ). Remarkably higher number of haplotypes (659 out of 9576) were significantly affected at the level of the Spike protein. For the Delta variant, approximately equal numbers of haplotypes showed reduced (91 haplotypes) and enhanced (81 haplotypes) peptide presentation (Table S2). Situation was highly biased towards more effective presentation in the Omicron case: 453 and 100 haplotypes with the significant increase and decrease in numbers of tight binders, respectively.

Less than $10 \%$ of the identified haplotypes (54 out of 659 ) were present in the list of the most frequent HLA haplotypes. A*24:02-B*07:02-C*07:02 haplotype had the highest frequency: 1.2\% in Europe, 2.7\% in North America, 4\% in North-East Asia, 1.6\% in South Asia and 1.4\% in Moscow, Russia. Efficiency of viral peptide presentation by this set of HLA molecules was reduced by $20 \%$ and $33.3 \%$ for the Omicron and Delta variants, respectively.

Significant alterations in peptide presentation for HLA class II haplotypes were found only for the Spike protein case (Table S3). Surprisingly, these differences were completely opposite for the Delta and Omicron variants: 320 haplotypes were associated with enhanced presentation of Delta peptides, while 951 haplotypes were associated with reduced Omicron peptide presentation. Consistently with the individual allele-level analysis, all identified haplotypes contained HLA-DPA1*01:03/DPB $1 * 03: 01$ allele for the Delta variant and HLA-DRB1*03:01 allele for the Omicron variant.

There were only four frequent HLA class II haplotypes affected by the Delta mutations and six haplotypes for the Omicron variant (Table S3). From them, DRB1*03:01-DPB1*04:01DQB 1*02:01 had especially high worldwide frequency: 4.2\% in Europe, $10 \%$ in Western Asia and $10 \%$ in Moscow, Russia. Based on our computational analysis, the number of tight binding SARS-CoV-2 peptides for this haplotype is reduced by $33.3 \%$ in the Omicron variant compared to the reference Wuhan virus.

\section{Discussion} SARS-CoV-2 variants. First, the analysis was performed at the level of individual alleles. Only one allele with significantly altered peptide presentation was identified: HLA-B*35:03 had one 
more Omicron/Delta tight binding peptide in NSP12 protein. Thus, we predicted the complete absence of T-cell immunity evasion at the level of the whole virus. When the analysis was limited to the Spike protein, remarkably higher number of alleles was identified: 8 alleles showed enhanced ability of Omicron/Delta peptide presentation, while 4 remaining alleles showed significant decrease in the number of tight binders.

HLA-DRB1*03:01 molecule had the highest escape rate for the Omicron peptides: the only predicted tight binder from the Wuhan variant for this allele (PINLVRDLPQGFSAL) lost its binding affinity because of several mutations: N211 deletion, L212I substitution and EPE 212214 insertion. Aside from the high predicted binding affinity, CD4 T-cell immunogenicity of the mentioned peptide was previously validated in two experimental reports (Keller et al., 2020; Verhagen et al., 2021). The set of tight binding peptides for another allele, HLA-B*07:02, was also exhausted because of the Spike P681R mutation which was present both in the Omicron and Delta variants. In concordance with our findings, Hamelin with co-authors showed that epitopes associated with B07 supertype were likely to escape CD8 T-cell immunity during the first year of the pandemic (Hamelin et al., 2021).

Given the results of the allele-level analysis, we constructed theoretical libraries of HLA class I and II haplotypes composed of the considered alleles. Few haplotypes which included alleles with altered presentation of mutant peptides also showed significant differential peptide presentation. One of the identified HLA class II haplotypes had especially high worldwide frequency, including Europe, Western Asia and Moscow, Russia. Namely, the number of highly affine peptides for DRB1*03:01-DPB1*04:01-DQB1*02:01 was reduced by $33.3 \%$ in the Omicron variant compared both to the Wuhan and Delta. Individuals carrying this haplotype could possibly develop impaired CD4 T-cell response to the Omicron variant following Wuhan Spike protein-based vaccination, which would consequently imply impaired antibody response. Nevertheless, the overwhelming majority of haplotypes were not associated with significantly reduced Omicron/Delta peptide presentation, which fully agrees with the recently conducted experiments on small patient cohorts (GeurtsvanKessel et al., 2021; Keeton et al., 2021; May et al., 2021; Liu et al., 2022; Mazzoni et al., 2022).

\section{Conclusions}

The high diversity of HLA alleles and haplotypes coupled with the specificity of peptide presentation strongly limits the potential of T-cell immune response evasion of SARS-CoV-2. In this manuscript we identified several HLA class I and II alleles with impaired presentation of peptides originating from the Spike protein of the Omicron and Delta variants. The strongest effect was observed for HLA-DRB $1 * 03: 01$ allele, which lost all tightly binding peptides because of the Omicron mutations. At the same time, peptide presentation at the level of the whole virus was practically unaffected by the mutations. Given that we hypothesize that some individuals 
vaccinated with Spike protein-based vaccines could develop the impaired T-cell immune responses to the Omicron variant. Experimental verification of this hypothesis is warranted.

\section{Funding}

The research was performed within the framework of the Basic Research Program at HSE University (MS, AGal; study design, interpretation), the donation of SberBank to Faculty of Biology and Biotechnology at HSE University (SN, AT; bioinformatics analysis, T-CoV portal development) and the Russian Science Foundation (Project No. 17-74-30019; MZ, II, IK, AM, AGab; HLA/peptide experiments).

\section{References}

Cameroni E, Bowen JE, Rosen LE, Saliba C, Zepeda SK, Culap K, Pinto D, VanBlargan LA, De Marco A, di Iulio J, Zatta F, Kaiser H, Noack J, Farhat N, Czudnochowski N, HavenarDaughton C, Sprouse KR, Dillen JR, Powell AE, Chen A, Maher C, Yin L, Sun D, Soriaga L, Bassi J, Silacci-Fregni C, Gustafsson C, Franko NM, Logue J, Iqbal NT, Mazzitelli I, Geffner J, Grifantini R, Chu H, Gori A, Riva A, Giannini O, Ceschi A, Ferrari P, Cippà PE, Franzetti-Pellanda A, Garzoni C, Halfmann PJ, Kawaoka Y, Hebner C, Purcell LA, Piccoli L, Pizzuto MS, Walls AC, Diamond MS, Telenti A, Virgin HW, Lanzavecchia A, Snell G, Veesler D, Corti D. 2021. Broadly neutralizing antibodies overcome SARS-CoV-2 Omicron antigenic shift. Nature. DOI: 10.1038/s41586-021-04386-2.

Choo SY. 2007. The HLA System: Genetics, Immunology, Clinical Testing, and Clinical Implications. Yonsei Medical Journal 48:11. DOI: 10.3349/ymj.2007.48.1.11.

Elbe S, Buckland-Merrett G. 2017. Data, disease and diplomacy: GISAID's innovative contribution to global health. Global Challenges 1:33-46. DOI: 10.1002/gch2.1018.

GeurtsvanKessel CH, Geers D, Schmitz KS, Mykytyn AZ, Lamers MM, Bogers S, Gommers L, Sablerolles RSG, Nieuwkoop NN, Rijsbergen LC, van Dijk LLA, de Wilde J, Alblas K, Breugem TI, Rijnders BJA, de Jager H, Weiskopf D, van der Kuy PHM, Sette A, Koopmans MPG, Grifoni A, Haagmans BL, de Vries RD. 2021. Divergent SARS CoV-2 Omicron-specific T- and B-cell responses in COVID-19 vaccine recipients. medRxiv:2021.12.27.21268416. DOI: 10.1101/2021.12.27.21268416.

Gonzalez-Galarza FF, McCabe A, Santos EJM Dos, Jones J, Takeshita L, Ortega-Rivera ND, Cid-Pavon GMD, Ramsbottom K, Ghattaoraya G, Alfirevic A, Middleton D, Jones AR. 2020. Allele frequency net database (AFND) 2020 update: Gold-standard data classification, open access genotype data and new query tools. Nucleic Acids Research 48:D783-D788. DOI: 10.1093/nar/gkz1029.

Hamelin DJ, Fournelle D, Grenier J-C, Schockaert J, Kovalchik KA, Kubiniok P, Mostefai F, Duquette JD, Saab F, Sirois I, Smith MA, Pattijn S, Soudeyns H, Decaluwe H, Hussin J, Caron E. 2021. The mutational landscape of SARS-CoV-2 variants diversifies T cell targets in an HLA-supertype-dependent manner. Cell systems. DOI: 10.1016/j.cels.2021.09.013.

Keeton R, Tincho MB, Ngomti A, Baguma R, Benede N, Suzuki A, Khan K, Cele S, Bernstein M, Karim F, Madzorera S V, Moyo-Gwete T, Mennen M, Skelem S, Adriaanse M, Mutithu D, Aremu O, Stek C, Bruyn E du, Van Der Mescht MA, de Beer Z, de Villiers TR, Bodenstein A, van den Berg G, Mendes A, Strydom A, Venter M, Grifoni A, Weiskopf D, Sette A, Wilkinson RJ, Bekker L-G, Gray G, Ueckermann V, Rossouw T, Boswell MT, 

$\mathrm{T}$ cell responses induced upon vaccination or infection remain robust against Omicron. medRxiv:2021.12.26.21268380. DOI: 10.1101/2021.12.26.21268380.

Keller MD, Harris KM, Jensen-Wachspress MA, Kankate V V, Lang H, Lazarski CA, DurkeeShock J, Lee P-H, Chaudhry K, Webber K, Datar A, Terpilowski M, Reynolds EK, Stevenson EM, Val S, Shancer Z, Zhang N, Ulrey R, Ekanem U, Stanojevic M, Geiger A, Liang H, Hoq F, Abraham AA, Hanley PJ, Cruz CR, Ferrer K, Dropulic L, Gangler K, Burbelo PD, Jones RB, Cohen JI, Bollard CM. 2020. SARS-CoV-2-specific T cells are rapidly expanded for therapeutic use and target conserved regions of the membrane protein. Blood 136:2905-2917. DOI: 10.1182/blood.2020008488.

Kumar B V., Connors TJ, Farber DL. 2018. Human T Cell Development, Localization, and Function throughout Life. Immunity 48:202-213. DOI: 10.1016/j.immuni.2018.01.007.

Kyriakidis NC, López-Cortés A, González EV, Grimaldos AB, Prado EO. 2021. SARS-CoV-2 vaccines strategies: a comprehensive review of phase 3 candidates. npj Vaccines 6:28. DOI: 10.1038/s41541-021-00292-w.

Liu J, Chandrashekar A, Sellers D, Barrett J, Lifton M, McMahan K, Sciacca M, VanWyk H, Wu C, Yu J, Collier AY, Barouch DH. 2022. Vaccines Elicit Highly Cross-Reactive Cellular Immunity to the SARS-CoV-2 Omicron Variant. medRxiv:2022.01.02.22268634. DOI: $10.1101 / 2022.01 .02 .22268634$.

Mamedov A, Vorobyeva N, Filimonova I, Zakharova M, Kiselev I, Bashinskaya V, Baulina N, Boyko A, Favorov A, Kulakova O, Ziganshin R, Smirnov I, Poroshina A, Shilovskiy I, Khaitov M, Sykulev Y, Favorova O, Vlassov V, Gabibov A, Belogurov A. 2020. Protective Allele for Multiple Sclerosis HLA-DRB1*01:01 Provides Kinetic Discrimination of Myelin and Exogenous Antigenic Peptides. Frontiers in Immunology 10. DOI: 10.3389/fimmu.2019.03088.

May DH, Rubin BER, Dalai SC, Patel K, Shafiani S, Elyanow R, Noakes MT, Snyder TM, Robins HS. 2021. Immunosequencing and epitope mapping reveal substantial preservation of the T cell immune response to Omicron generated by SARS-CoV-2 vaccines. medRxiv:2021.12.20.21267877. DOI: 10.1101/2021.12.20.21267877.

Mazzoni A, Vanni A, Spinicci M, Capone M, Lamacchia G, Salvati L, Coppi M, Antonelli A, Carnasciali A, Farahvachi P, Giovacchini N, Aiezza N, Malentacchi F, Zammarchi L, Liotta F, Rossolini GM, Bartoloni A, Cosmi L, Maggi L, Annunziato F. 2022. SARS-CoV-2 Spike-Specific CD4+ T Cell Response Is Conserved Against Variants of Concern, Including Omicron. Frontiers in Immunology 13. DOI: 10.3389/fimmu.2022.801431.

Nelde A, Bilich T, Heitmann JS, Maringer Y, Salih HR, Roerden M, Lübke M, Bauer J, Rieth J, Wacker M, Peter A, Hörber S, Traenkle B, Kaiser PD, Rothbauer U, Becker M, Junker D, Krause G, Strengert M, Schneiderhan-Marra N, Templin MF, Joos TO, Kowalewski DJ, Stos-Zweifel V, Fehr M, Rabsteyn A, Mirakaj V, Karbach J, Jäger E, Graf M, Gruber L-C, Rachfalski D, Preuß B, Hagelstein I, Märklin M, Bakchoul T, Gouttefangeas C, Kohlbacher O, Klein R, Stevanović S, Rammensee H-G, Walz JS. 2021. SARS-CoV-2-derived peptides define heterologous and COVID-19-induced T cell recognition. Nature Immunology 22:7485. DOI: $10.1038 / \mathrm{s} 41590-020-00808-\mathrm{x}$.

Nersisyan S, Zhiyanov A, Shkurnikov M, Tonevitsky A. 2022. T-CoV: a comprehensive portal of HLA-peptide interactions affected by SARS-CoV-2 mutations. Nucleic Acids Research 50:D883-D887. DOI: 10.1093/nar/gkab701.

Reynisson B, Alvarez B, Paul S, Peters B, Nielsen M. 2020. NetMHCpan-4.1 and 
NetMHCIIpan-4.0: improved predictions of MHC antigen presentation by concurrent motif deconvolution and integration of MS MHC eluted ligand data. Nucleic Acids Research 48:W449-W454. DOI: 10.1093/nar/gkaa379.

Schäfer C, Schmidt AH, Sauter J. 2017. Hapl-o-Mat: open-source software for HLA haplotype frequency estimation from ambiguous and heterogeneous data. BMC Bioinformatics 18:284. DOI: $10.1186 / \mathrm{s} 12859-017-1692-\mathrm{y}$.

Sekine T, Perez-Potti A, Rivera-Ballesteros O, Strålin K, Gorin J-B, Olsson A, Llewellyn-Lacey S, Kamal H, Bogdanovic G, Muschiol S, Wullimann DJ, Kammann T, Emgård J, Parrot T, Folkesson E, Rooyackers O, Eriksson LI, Henter J-I, Sönnerborg A, Allander T, Albert J, Nielsen M, Klingström J, Gredmark-Russ S, Björkström NK, Sandberg JK, Price DA, Ljunggren H-G, Aleman S, Buggert M, Akber M, Berglin L, Bergsten H, Brighenti S, Brownlie D, Butrym M, Chambers B, Chen P, Jeannin MC, Grip J, Gomez AC, Dillner L, Lozano ID, Dzidic M, Tullberg MF, Färnert A, Glans H, Haroun-Izquierdo A, Henriksson E, Hertwig L, Kalsum S, Kokkinou E, Kvedaraite E, Loreti M, Lourda M, Maleki K, Malmberg K-J, Marquardt N, Maucourant C, Michaelsson J, Mjösberg J, Moll K, Muva J, Mårtensson J, Nauclér P, Norrby-Teglund A, Medina LP, Persson B, Radler L, Ringqvist E, Sandberg JT, Sohlberg E, Soini T, Svensson M, Tynell J, Varnaite R, Kries A Von, Unge C. 2020. Robust T Cell Immunity in Convalescent Individuals with Asymptomatic or Mild COVID-19. Cell 183:158-168.e14. DOI: 10.1016/j.cell.2020.08.017.

Shiehzadegan S, Alaghemand N, Fox M, Venketaraman V. 2021. Analysis of the Delta Variant B.1.617.2 COVID-19. Clinics and Practice 11:778-784. DOI: 10.3390/clinpract11040093.

Shkurnikov M, Nersisyan S, Jankevic T, Galatenko A, Gordeev I, Vechorko V, Tonevitsky A. 2021. Association of HLA Class I Genotypes With Severity of Coronavirus Disease-19. Frontiers in Immunology 12. DOI: 10.3389/fimmu.2021.641900.

Verhagen J, van der Meijden ED, Lang V, Kremer AE, Völkl S, Mackensen A, Aigner M, Kremer AN. 2021. Human CD4+ T cells specific for dominant epitopes of SARS-CoV-2 Spike and Nucleocapsid proteins with therapeutic potential. Clinical and experimental immunology 205:363-378. DOI: 10.1111/cei.13627.

Zhao H, Lu L, Peng Z, Chen L-L, Meng X, Zhang C, Ip JD, Chan W-M, Chu AW-H, Chan K-H, Jin D-Y, Chen H, Yuen K-Y, To KK-W. 2022. SARS-CoV-2 Omicron variant shows less efficient replication and fusion activity when compared with Delta variant in TMPRSS2expressed cells. Emerging Microbes \& Infections 11:277-283. DOI: 10.1080/22221751.2021.2023329. 


\section{Figures}

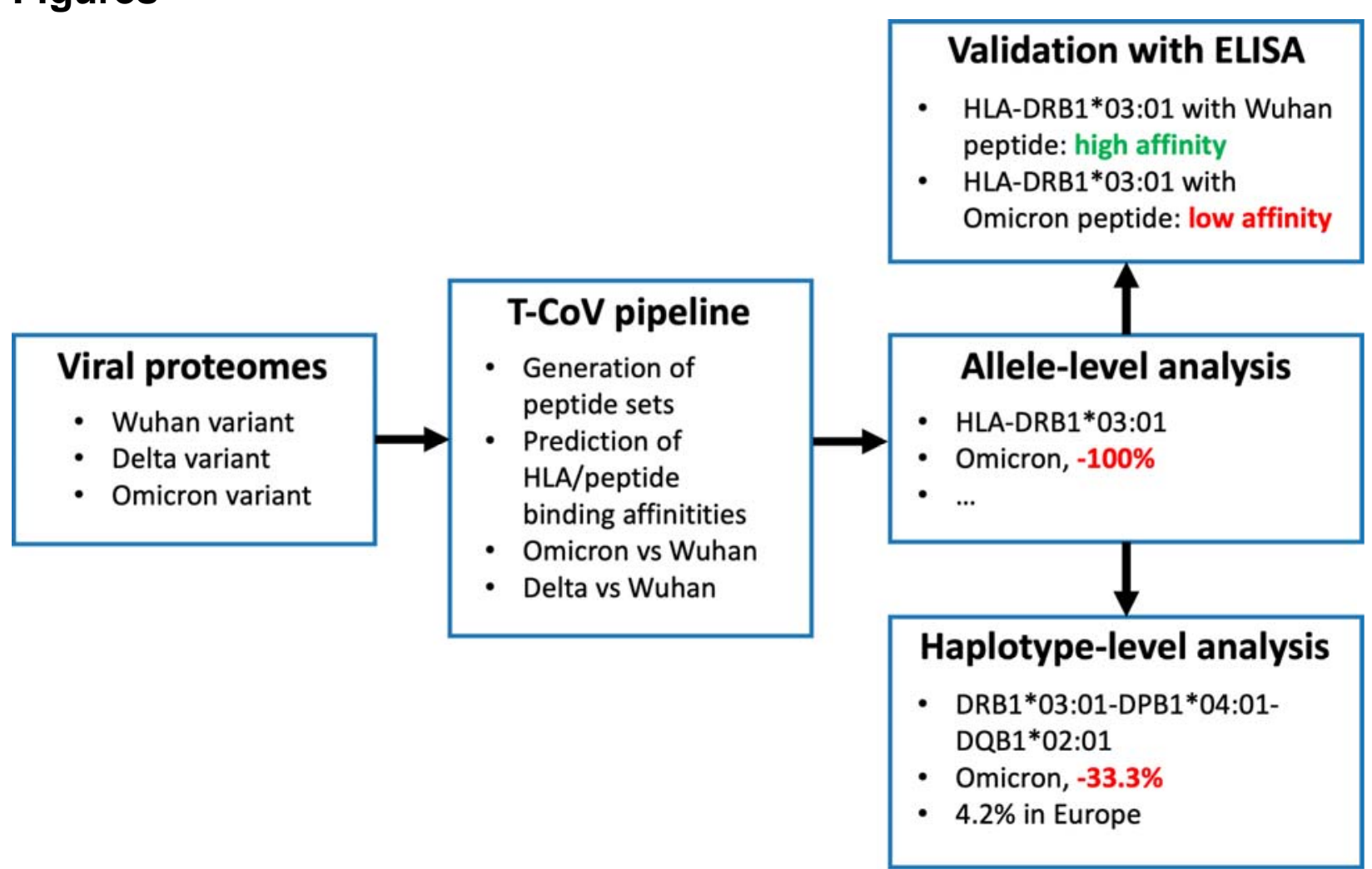

Figure 1. Workflow of the analysis. 


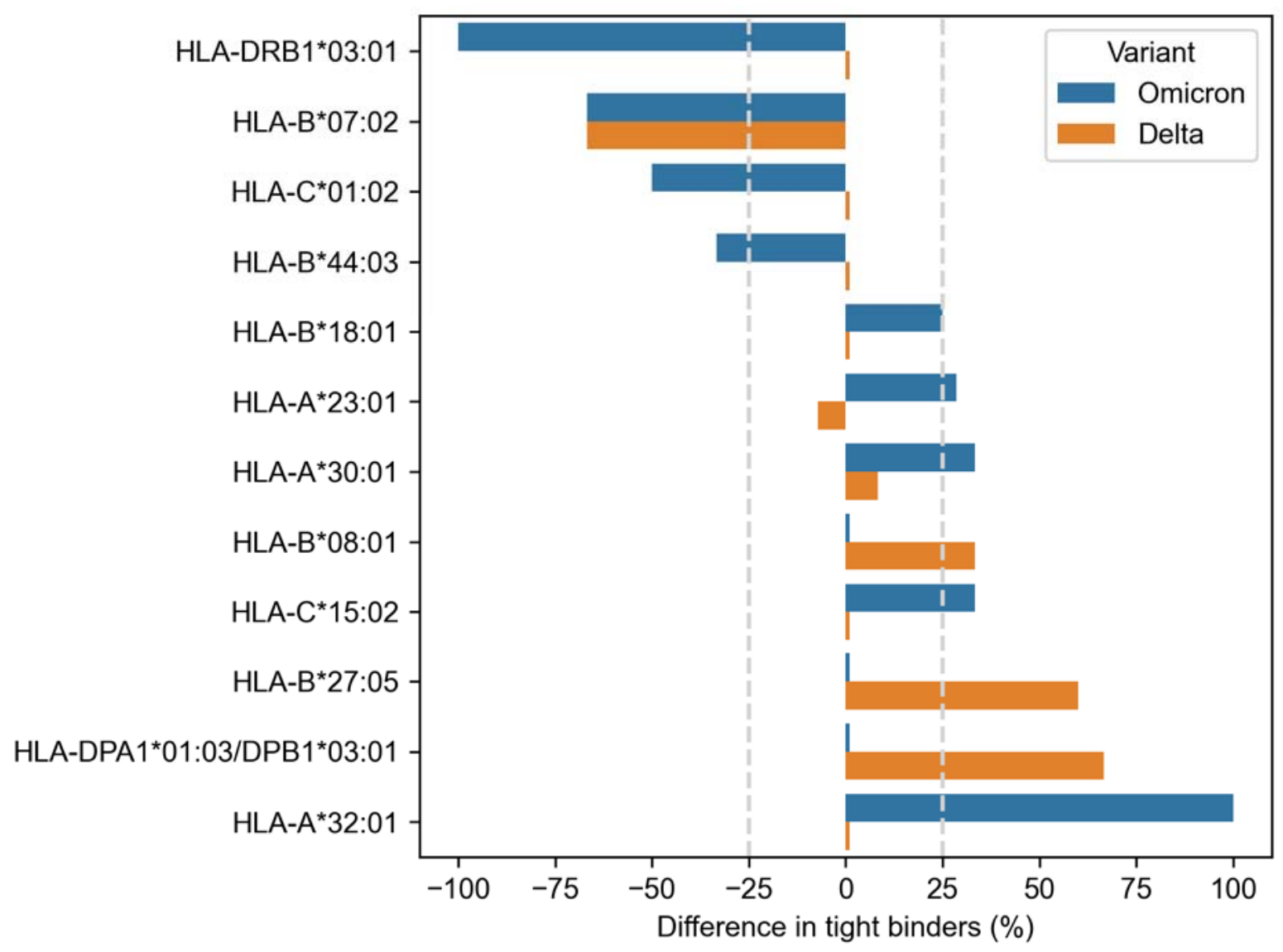

Figure 2. Differences in viral peptide presentation for the Delta and Omicron variants. The 


\section{A}

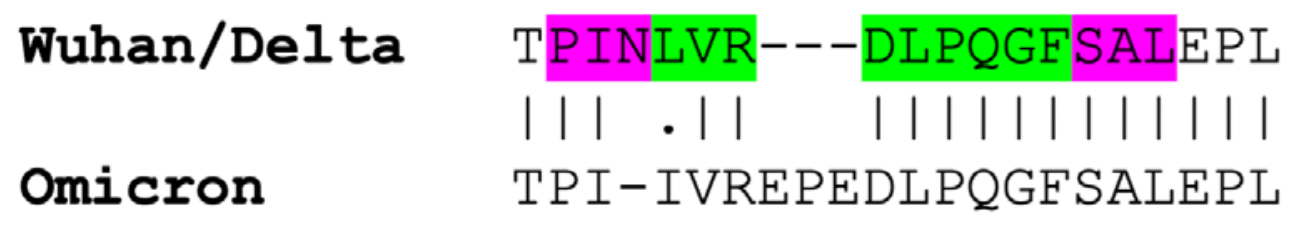

\section{B}

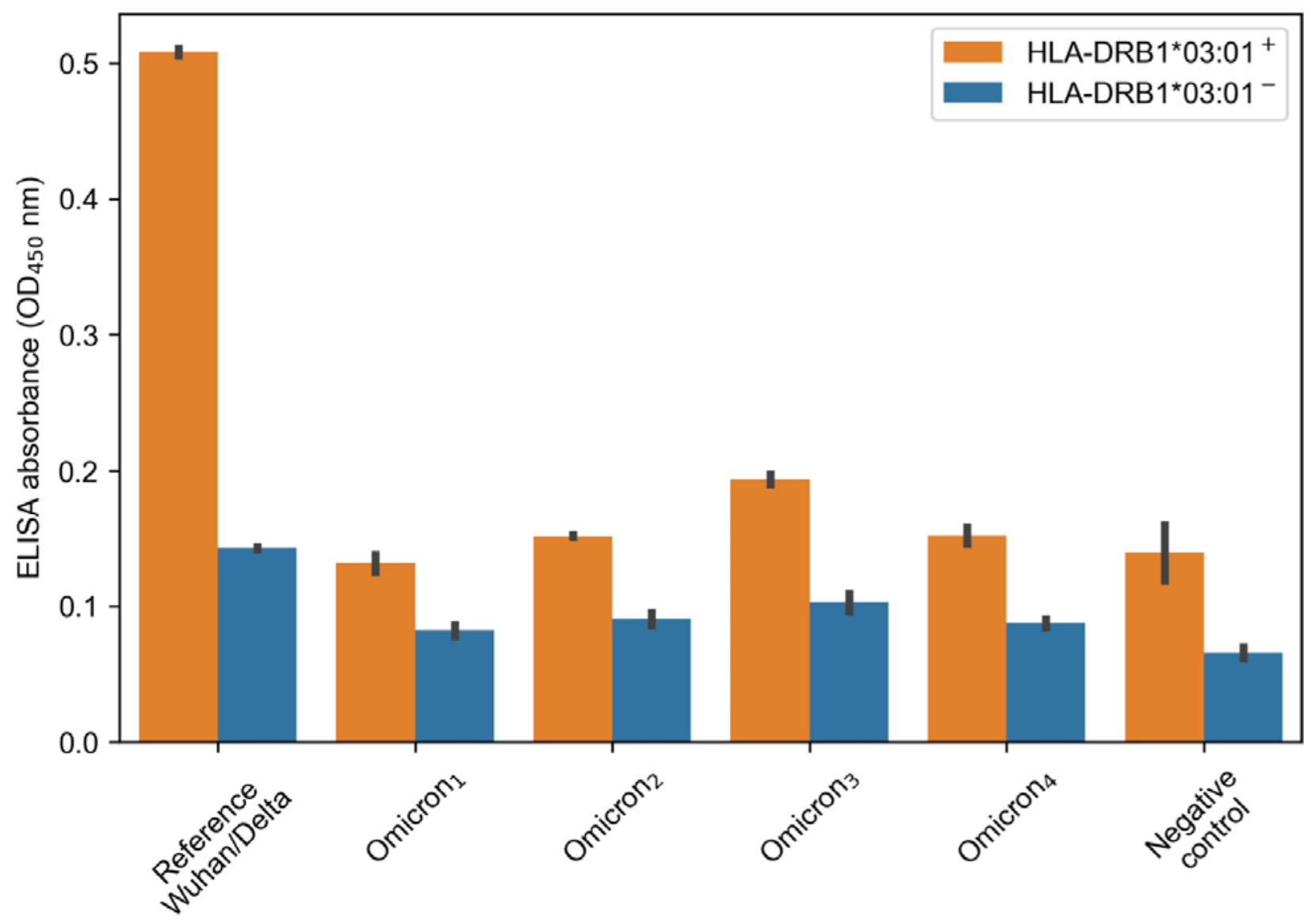

Thioredoxin-fused peptides binders. (A) The region of consideration. Green color stands for HLA-DRB1*03:01 9-mer analysis. Omicron 1, Omicron $_{2}$, Omicron $_{3}$, Omicron $_{4}$ stand for TPIIVREPEDLPQGF, IIVREPEDLPQGFSA, VREPEDLPQGFSALE and EPEDLPQGFSALEPL peptides. Negative control stands for the substrate construct, carrying only thioredoxin with the linker. 


\section{A}

\section{Haplotype-level analysis}

This tool allows one to compare viral peptide presentation by a given list of HLA molecules between two SARS-CoV-2 variants.

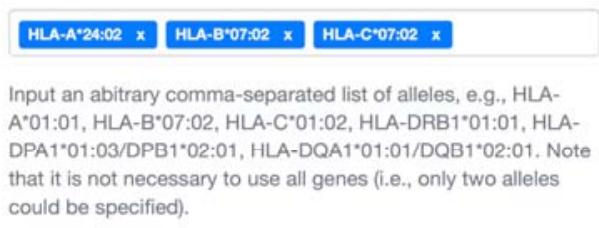

\section{Omicron GR/484A (B.1.1."}

Wuhan-Hu-1

Submit

Figure 4. The web interface of T-CoV new tool. (A) User can select HLA class I/II alleles and SARS-CoV-2 variants. (B) The results of analysis are grouped by viral proteins and entered alleles.

B

$$
\mathrm{T}-\mathrm{CoV}
$$
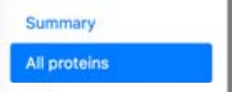

Spike

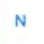

M

E

NS3

NS6

NS7a

NS7b

NS8

NS9b

NS9c

NSP1

NSP2

NSP3

NSP4

NSP5
Omicron GR/484A (B.1.1.529) vs Wuhan-Hu-1

\section{All proteins}

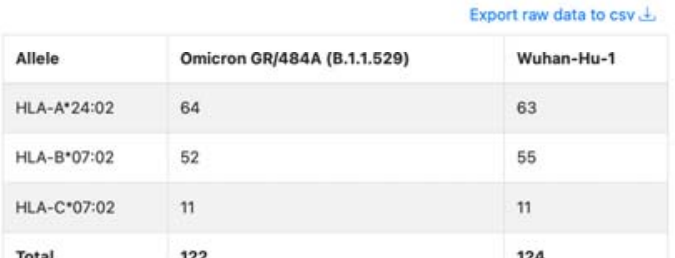

Total

122

Spike

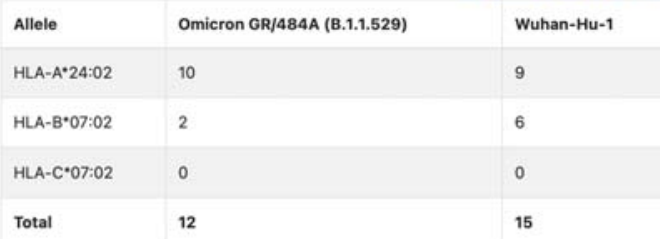

\section{Supplemental Information}

Table S1. The numbers of tightly binding Omicron/Delta/Wuhan peptides for individual HLA class I/II alleles.

Table S2. The numbers of tightly binding Omicron/Delta/Wuhan peptides for HLA class I haplotypes.

Table S3. The numbers of tightly binding Omicron/Delta/Wuhan peptides for HLA class II haplotypes. 